\title{
A Novel State of Charge Estimation for Energy Storage Systems Based on the Joint NARX Network and Filter Algorithm
}

\author{
Huan Li, Chuanyun Zou ${ }^{1, *}$, Carlos Fernandez ${ }^{2}$, Shunli Wang ${ }^{1}$, Yongcun Fan ${ }^{1}$, Donglei Liu ${ }^{1}$ \\ ${ }^{1}$ School of Information Engineering, Southwest University of Science and Technology, Mianyang \\ 621010, China; \\ ${ }^{2}$ School of Pharmacy and Life Sciences, Robert Gordon University, Aberdeen AB10-7GJ, UK. \\ *E-mail: zouchuanyun@swust.edu.cn
}

Received: 8 August 2021 / Accepted: 17 September 2021 / Published: 10 November 2021

\begin{abstract}
Lithium-ion batteries have the advantage of high energy density, low self-discharge rate, and long cycle life, and are currently the most widely used energy storage carriers. Accurate state of charge (SOC) estimation is essential to ensure the lithium-ion battery's safe and reliable operation. In order to improve the accuracy of estimation, this paper creatively applies the extended Kalman filter (EKF) to the improved nonlinear autoregressive algorithm with an exogenous neural network (NARXNN), forming a NARX-EKF neural network model for SOC prediction of the lithium-ion battery for the first time. This method avoids complicated equivalent modeling and parameter identification, rather directly maps the measured voltage, current, and temperature to the SOC. The data set is obtained by simulating the driving cycle load of the lithium-ion battery under different working conditions, and the network is tested under cyclic working conditions, dynamic working conditions, different temperature conditions, and different aging cycles. The SOC estimation results of the NARX-EKF model are evaluated from three aspects: mean absolute error (MAE), root mean square error (RMSE), and SOC error. Under cyclic conditions, the RMSE and MAE of NARXNN are only $1.4 \%$ and $1.3 \%$, which is only $50 \%$ of other neural networks. In the dynamic working condition test, the maximum error of NARXNN optimized by EKF is reduced by about $50 \%$, and the RMSE and MAE of the model are only $20 \%$ of other neural networks. When the ambient temperature changes, the RMSE and MAE of the model under low-temperature conditions were $1.2 \%$ and $0.9 \%$ respectively. The RMSE and MAE of the model under high-temperature conditions were $0.6 \%$ and $0.5 \%$ respectively. In addition, the NARX-EKF network can well solve the impact of different aging degrees of lithium-ion batteries on SOC estimation. When the battery health status is only $70 \%$, the RMSE and MAE of the model were only $2.7 \%$ and $2.5 \%$ respectively. The results show that the NARX-EKF model has high accuracy, robustness, and good application prospects.
\end{abstract}

Keywords: Lithium-ion Battery; Nonlinear Autoregressive Neural Network; State-of-Charge; Extended Kalman Filter

$\underline{\text { FULL TEXT }}$ 
(C) 2021 The Authors. Published by ESG (www.electrochemsci.org). This article is an open access article distributed under the terms and conditions of the Creative Commons Attribution license (http://creativecommons.org/licenses/by/4.0/). 